\title{
Production of a chlorophyll deficient streptomycin resistant mutant of Nicotiana tabacum for somatic hybridisation studies
}

\author{
Seiichi Toki and \\ Toshiaki Kameya
}

Institute for Agricultural Research, Tohoku

University, Sendai 980 , Japan

In order to produce a double mutant, sexual crosses between a streptomycin resistant mutant of Nicotiana tabacum (SR1) and a chlorophyll-deficient mutant of $N$. tabacum (Albino, A1) were carried out. From their offspring of this cross, a double mutant (SA) was selected and characterised with respect to streptomycin resistance and the ability to synthesize chlorophyll. Calli initiated from SA showed resistance to streptomycin and the chlorophyll content in SA leaves was lower than that of the normal green plants. Therefore, it is expected that the double mutant established here will be useful in studies of somatic hybridisation. Somatic hybrids between any other normal plants and the SA can be selected effectively by the appearance of green plants on a selective medium containing streptomycin.

\section{INTRODUCTION}

Recently, several somatic hybrids have been obtained by using various selection markers. In particular, resistant to amino acid analogs, antibiotics resistance or chlorophyll deficiency have been used as useful selection markers. However, if both positive and negative selection markers were incorporated into a plant, such a plant is called a universal hybridiser, since it can be hybridised with any wild type plants. Such double mutant have been reported in Daucus (LoSchivao et al., 1983) and in Nicotiana (Hamill et al., 1983; Pental et al., 1985).

In this study, we have sexually crossed between a streptomycin resistant Nicotiana tabacum SR1 (Maliga et al., 1973, 1975) and a chlorophyll deficient $N$. tabacum var. Hicks to produce a universal hybridiser; the double mutant so obtained was characterised with respect to streptomycin resistance and the ability to synthesize chlorophyll.

\section{MATERIALS AND METHODS}

In this study a streptomycin resistant mutant of $N$. tabacum, SR1 (Maliga et al., 1973, 1975), and the chlorophyll deficient mutant of N. tabacum, Albino (A1) var. Hicks obtained in our laboratory as a spontaneous mutant and grafted on wild type $N$. tabacum, were used as plant materials.

\section{Selection of double mutant plants: Anther culture}

Before sexual crosses between SR1 and Al were made we checked about inheritance of the albino trait. For this purpose, $\mathrm{Al}$ and the wild type Hicks were reciprocally crossed, and culture of anther from F1 hybrids was carried out using Nitsch's medium (Nitsch and Nitsch, 1969) supplemented with $1.5 \mathrm{mg} / 1 \mathrm{IAA}, 25 \mathrm{~g} / 1$ sucrose and $8 \mathrm{~g} / \mathrm{l}$ agar.

\section{Production of the SA double mutant}

In sexual crosses between $\mathrm{SR} 1$ and $\mathrm{Al}, \mathrm{SR} 1$ was used as the female parent to ensure transmission of the streptomycin resistant chloroplasts. To allow segregation of chlorophyll deficient genotypes, F1 plants were self-fertilised. Seeds from the selfed F1 plants were surface sterilised with 2 per cent $(W / V)$ sodium hypochlorite for $15 \mathrm{~min}$ followed by rinsing in sterile water. The seeds were germinated on hormone free MS medium (Murashige and Skoog, 1962) solified with $8 \mathrm{~g} / \mathrm{l}$ agar, under continuous fluorescent light $(3000 \mathrm{~lx})$ at $25^{\circ} \mathrm{C}$.

The albino plants emerging in the $\mathrm{F} 2$ generation were expected to be double mutants (streptomycin resistant albino $N$. tabacum: designated as SA) and they were grafted on wild type $N$. tabacum. 


\section{Growth test}

The stability of streptomycin resistance was studied in seedlings and calli.

Seeds from SR1, Al, SA and wild type $N$. tabacum var. Xanthi were germinated on filter paper soaked with water with $0-1000 \mathrm{mg} / 1$ streptomycin sulfate under continuous fluorescent light $(3000 \mathrm{~lx})$ at $25^{\circ} \mathrm{C}$. After 14 days from planting, growth rate of the roots and colour of the cotyledons were determined.

Callus was aseptically initiated from whole seeds and subcultured once on MS medium with $1 \mathrm{mg} / 1$ 2,4-D (2,4-dichlorophenoxyacetic acid) and $0 \cdot 1 \mathrm{mg} / 1 \mathrm{kinetin}$ solidified with $8 \mathrm{~g} / 1$ agar. The viable callus mass was transferred into a MS liquid medium (above medium minus agar with MS vitamins were substituted for UM vitamines (Uchimiya and Murashige, 1974), then subcultured for at least ten passages before using for growth studies. Uniform volumes of callus of SA, $\mathrm{Al}$ and Xanthi (wild type) were placed on MS medium containing $1 \mathrm{mg} / 1 \quad 2,4-\mathrm{D}$ and $0.1 \mathrm{mg} / 1$ kinetin, $0-10000 \mathrm{mg} / \mathrm{l}$ streptomycin sulfate and $8 \mathrm{~g} / 1$ agar. After 4 weeks, the fresh weight of the calli was determined.

\section{Measurement of chlorophyll content}

The surface sterilised seeds from SA plants were cultured on hormone free MS medium containing $8 \mathrm{~g} / \mathrm{l}$ agar at 15,25 and $35^{\circ} \mathrm{C}$ under continuous fluorescent light $(30001 \mathrm{x})$. The SR1 seeds were cultured at $25^{\circ} \mathrm{C}$ as control. Chlorophyll was extracted from leaves with 80 per cent acetone at 100 days after culture and determined according to Arnon's method (Arnon, 1949).

\section{RESULTS}

\section{Inheritance of the albino trait}

The F1 hybrids of reciprocal crosses between $\mathrm{Al}$ and Hicks (wild type) were all green plants. There- fore, the albino trait was due to recessive nuclear genes.

The results obtained from anther culture of the Fl hybrids are shown in table 1. The deviations of observed from expected ratios were not significant at 5 per cent level. These results show that expression of the albino trait is due to recessive mutations at two loci.

The progeny of crosses between SR1 and Al were also all green plants. F1 hybrids were selfed and the progeny were scored for green plants: albino plants segregation. The results shown in table 2 confirm our expectation that expression of albino trait resulting from anter culture was subject to two recessive genes. The albino plants that emerged in the F1 progeny were expected to be double mutants SA with respect to albinism and streptomycin resistance.

\section{Characterisation of SA: Growth test}

The results of growth tests for streptomycin resistance of seedlings are shown in fig. 1. Increasing concentrations of streptomycin caused a decrease in the radicle length. But rate of decrease in length with streptomycin concentration in SR1 and SA was smaller than in Xanthi. However, the most striking effect of streptomycin was appeared on the colour of the cotyledons (table 3 ). In green plants, even at $100 \mathrm{mg} / 1$ streptomycin, the cotyledons of sensitive seedlings (Xanthi) turned yellow. On the other hand, cotyledons of resistant seedlings (SR1) remained still green. A similar phenomenon was observed in albino plants. Without streptomycin, both $\mathrm{Al}$ and SA germinated normally and the colour of the cotyledons were cream colour. But at $100 \mathrm{mg} / 1$ streptomycin, the colour of A1 turned white, while the cotyledons of SA were still cream coloured. Fig. 2 shows the results of growth test of calli. The relative increase in fresh weight expressed as percentage of A1 and Xanthi were much affected than those of SA by streptomycin. Even at $10000 \mathrm{mg} / 1$ streptomycin, SA callus could grow.

Table 1 The results of another culture of $A l X$ Hicks $\left(F_{1}\right)$ plants

\begin{tabular}{|c|c|c|c|c|c|c|}
\hline \multirow[b]{2}{*}{ Cross } & \multirow{2}{*}{$\begin{array}{l}\text { No. of } \\
\text { incubated } \\
\text { anthers }\end{array}$} & \multicolumn{2}{|c|}{$\begin{array}{l}\text { No. of regenerated } \\
\text { plantlets }\end{array}$} & \multicolumn{2}{|c|}{ Expected segregation } & \multirow[b]{2}{*}{$\chi^{2}$ at $p=0.05$} \\
\hline & & green & albino & green & albino & \\
\hline Al $\times$ Hicks & 335 & 49 & 33 & 3 & 1 & $0.3>x^{2}>0.2$ \\
\hline Hicks $\times \wedge 1$ & 560 & 116 & 35 & 3 & 1 & $0.7>x^{2}>0.5$ \\
\hline SR1 & 555 & 220 & 0 & 1 & 0 & \\
\hline
\end{tabular}


Table 2 The results of crosses between SR $1 \times \mathrm{Al}$

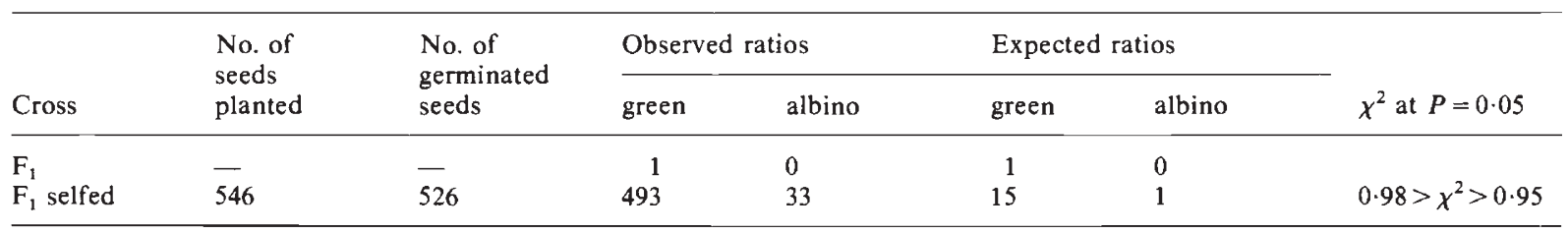

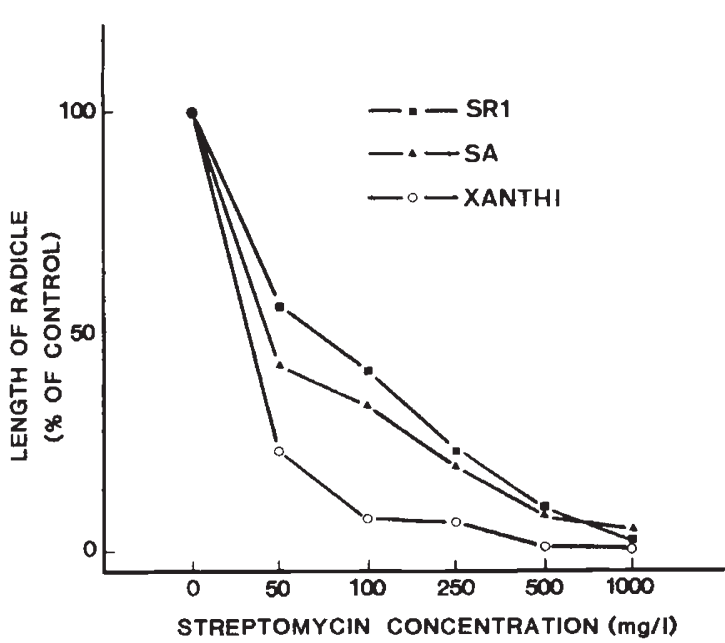

Figure 1 The effect of streptomycin concentration on the radicle length of germinating seedlings of various lines after 14 days of culture.

Table 3 The effect of streptomycin concentrations on the colour of the cotyledons

\begin{tabular}{lllllll}
\hline $\begin{array}{l}\text { Streptomycin (mg/l) } \\
\text { Line }\end{array}$ & 0 & 50 & 100 & 250 & 500 & 1000 \\
\hline Xanthi (wild type) & G & YG & Y & Y & W & W \\
SR1 & G & G & G & G & YG & YG \\
Al & C & W & W & W & W & W \\
SA & C & C & C & C & W & W \\
\hline
\end{tabular}

The colour of the cotyldons: C, Cream; G, Green, W, White; Y, Yellow; YG, Yellowish green.

Table 4 Chlorophyll content of SR1 and SA leaves grown at different temperatures

\begin{tabular}{llll}
\hline Line & $\begin{array}{l}\text { Temp. } \\
\left({ }^{\circ} \mathrm{C}\right)\end{array}$ & $\begin{array}{l}\mathrm{Chl} \\
(\mathrm{mg} / \mathrm{gFW})\end{array}$ & $\begin{array}{l}\text { Relative } \\
\text { values }\end{array}$ \\
\hline SR1 & 25 & 1.831 & 1 \\
SA & 15 & 0.008 & 0.004 \\
SA & 25 & 0.431 & 0.235 \\
SA & 35 & 0.081 & 0.044
\end{tabular}

Chl, chlorophyll; FW, fresh weight

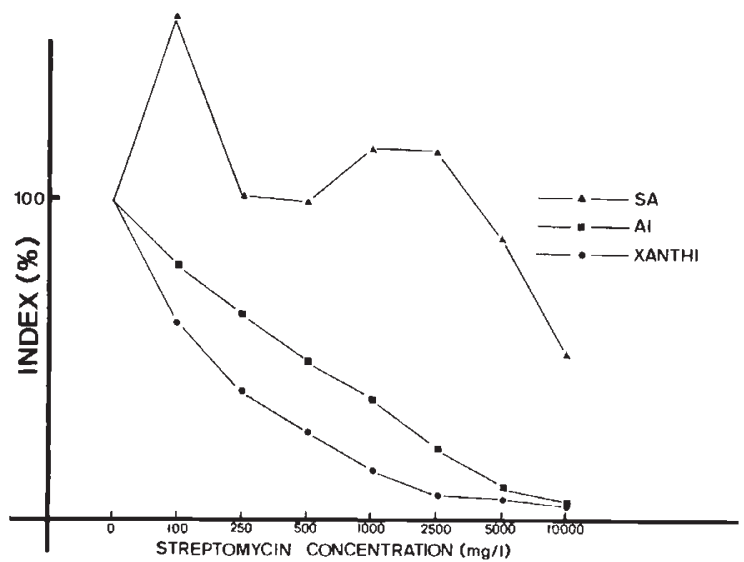

Figure 2 The effect of streptomycin concentration on the growth of callus after 4 weeks of culture.

\section{Measurement of chlorophyll contents}

The chlorophyll content of SA seedling were dependent upon temperature (table 4). This showed maximum at $25^{\circ} \mathrm{C}$ and decreased at either $15^{\circ} \mathrm{C}$ or $35^{\circ} \mathrm{C}$. Compared with the chlorophyll content in SR1, the content of SA was only 20 per cent even at $25^{\circ} \mathrm{C}$. So the leaf colour of SA was clearly distinguishable from that of normal green plants by the naked eye.

\section{DISCUSSION}

Both streptomycin resistance and chlorophyll deficiency have been utilized as markers for selecting or identifying somatic hybirds in cell fusion studies. By sexual crossing, we have combined these two traits in a single plant and studied the inheritance and stability of the albino trait as well as the property of streptomycin resistance in SA. The albino trait is determined by two recessive genes as in another chlorophyll deficient mutant of $N$. tabacum, Red Russian Ws (Clausen and Cameron, 1950; Moav and Cameron, 1950). The expression of the albino trait was also shown to be dependent upon temperature, with the maximum chlorophyll contents at $25^{\circ} \mathrm{C}$. The leaf 
colour of SA, however, was clearly distinguishable from that of normal green plants to the eye so that temperature dependence of SA expression did not disturb hybrid selection. The resistance of SA to streptomycin was comparable with that of the SR1 parent. Furthermore, this double mutant has a high regeneration capacity (data not shown).

These results indicate that SA mutant will be useful in studies of somatic hybridisation. The somatic hybrids should be green and capable of regenerated on a medium containing streptomycin.

Acknowledgements We thank Professor H. Suge for helpful suggestions and $\mathrm{Mr} \mathrm{H}$. Tokairin for technical assistance.

\section{REFERENCES}

ARNON, D. 1. 1949. Copper enzymes in isolated chloroplasts. Polyphenoloxidase in Beta vulgaris. Plant Physiol., 24, 1-15.

CLAUSEN, R. E. AND CAMERON, D. R. 1950. Inheritance in Nicotiana tabacum. XXIII. Duplicate factors for chlorophyll production. Genetics, 35, 4-10.
HAMILL, J. D., PENTAL, D,, ( OC:KING, F. C. ANI) MÜLLER, A. 3. 1983. Production of a nitrate reductase deficient streptomycin resistant mutant of Nicotiana tabacum for somatic hybridisation studies. Heredity, 50, 197-200.

LOSCHIVO, F., GIOVINAZZO, G. AND TERZI, M. 1983, 8 Azaguanine resistant carrot cell mutants and their use as universal hybridizers. Mol. Gen. Genet., 192, 326-329.

MALIGA, P., SZ.-BREZNOVITS, Á. AND MARTON, L. 1973. Streptomycin-resistant plants from callus culture of haploid tobacco. Nature New Biol., 244, 29-30.

MAI.I(JA, P., SZ.-BREZNOVITS, A., MARTON, L. ANI) JOO, F: 1975. Non-Mendelian streptomycin-resistant tobacco mutant with altered chloroplasts and mitochondria. Nature, 255, 401-402.

MOAV, R. AND CAMERON, D. R. 1960. I. The expression of instability in $N$. tabacum X N. plumbaginifolia. Am. J. Bot., 47, 87-93.

MURASHIGE, T. AND SKOO(j, F. 1962. A revised medium for rapid growth and bio assays with tobacco tissue cultures. Physiol. Plant., 15, 473-497.

NITSCH, J. P. AND NITSCH, C. 1969. Haploid plants from pollen grains. Science, $163,85-87$

PENTAL, D., HAMII.L, J. I). AND (COCKING, E. C. 1984. Somatic hybridization using a double mutant of Nicotiana tabacum. Heredity, 53, 79-83.

UCHIMIYA, H. ANI) MURASHI(jE, T. 1974. Evaluation of parameters in the isolation of viable protoplasts from cultured tobacco cells, Plant Physiol., 54, 936-944. 